\title{
Attenuation Performance of a Semi-Active Helmholtz Resonator in a Grazing Flow Duct
}

\author{
Xiaofeng Shi ${ }^{1}$, Cheuk Ming Mak ${ }^{1}$, Jun Yang ${ }^{2}$ \\ ${ }^{1}$ Department of Building Services Engineering, The Hong Kong Polytechnic University, Hong Kong, China \\ ${ }^{2}$ Key Laboratory of Acoustics, Institute of Acoustics, Chinese Academy of Sciences, Beijing, China \\ Email: cheuk-ming.mak@polyu.edu.hk
}

Received December 24, 2012; revised January 25, 2013; accepted February 7, 2013

\begin{abstract}
Side branch Helmholtz resonators (HRs) are widely used to control low frequency tonal noise in air duct system. The passive Helmholtz resonator only works effectively over a narrow frequency range around resonance frequency. Changes in the exciting frequency and temperature will decrease the noise reduction performance. Many studies have been conducted on incorporating a Helmholtz resonator with active noise control to tuning the resonance frequency of HRs. The objective of this study is to study the effect of flow on the semi active Helmholtz resonator for duct noise control. Owing to a low Mach number air flow, the discontinuity condition at the joint is analytically formulated according to the conservation of the momentum and mass of air flow. Based on the transfer function at the junction, a controller function is proposed to tune the semi-active Helmholtz resonator under flow condition.
\end{abstract}

Keywords: Helmholtz Resonator; Flow Effect; Semi-Active Control

\section{Introduction}

Helmholtz resonators are often used in duct system to control low frequency noise. It consists of a small neck and a cavity. The resonator yields high transmission loss performance at its resonance frequency. However, the effective frequency range of Helmholtz resonator is very narrow and limited and HRs are often designed according to the exciting frequency of source. Changes in exciting frequency and temperature result in the inconsistent between the noise source and HRs and hence decrease the effectiveness of the HRs.

In order to adapt to the changes, a solution is integrating the Helmholtz resonator with active control system. Such system is referred to as a semi active Helmholtz resonator. Semi active control method utilizes active noise control strategy to tune the resonance frequency of passive HRs. Many studies were focused on tuning the resonance frequency via changing the volume of the cavity or the neck area [1-5]. Although the resonance frequency can be changed through the semi-active control, the adaptive resonators still work effectively over the natural resonance frequency and the effective frequency range is still narrow. If it is necessary to control relative low frequency noise, the tunable mechanical structure needs to be significantly complex and bulky. Later, some researchers started to extend the efficient frequency range of the resonator with the termination impedance control. Radcliffe and Birdsong [6] proposed an electronically tuned semi-active Helmholtz resonator. They controlled the acoustic impedance of the resonator through mounting a loudspeaker at the end of the HR. The control source was stimulated by a closed-loop adaptive control strategy to change the resonance frequency. Utsumi [7] also investigated a side branch Helmholtz resonator with active termination impedance. He explored the possibility of broad band noise control through varying the termination impedance. Based on the derived controller transfer function, he demonstrated the efficiency of the method by computer simulation. Yuan [8] proposed to control it with positive real impedance to make the active HR more reliable and robust.

Flow generated produced by the ventilation fans influences acoustic impedance of the Helmholtz resonator when flow passes through the orifice. The sound absorption performance of the resonator decreases as a result of the affected impedance. Meyer et al. [9] studied the flow effect on acoustic impedance and their experiments showed that the resonance frequency shifted to a higher frequency with the rising flow speed and a minimum attenuation even a negative value occurred above the resonance frequency. Anderson [10] measured the acoustic impedance of a single side branch HR in a circular flow duct and found that fundamental frequency increased with increasing flow while neck end correction decreased. Hersch and walker [11] derived a semi em- 
pirical model for a HR mounted in a grazing flow duct. Their result showed that the resistance increased linearly as the flow velocity increased.

Previous work on the semi active control with Helmholtz resonator did not take into account the effect of the mean flow. This paper aims at studying the effect of flow on the semi active Helmholtz resonator for duct noise control. Firstly, the plane wave propagation in a flow duct is introduced and the discontinuity condition is analytically derived. Then, the control controller transfer function under flow condition is proposed.

\section{Plane Wave Propagation}

When a plane wave propagates in a mean flow duct, the wave equation is [12]:

$$
\left(1-M^{2}\right) \frac{\partial^{2} p}{\partial x^{2}}+k^{2} p-2 j k M \frac{\partial p}{\partial x}=0
$$

$M$ denotes the Mach number $M=u_{0} / c$. The solution to the wave equation is:

$$
\begin{aligned}
& p(x)=p^{+} \mathrm{e}^{-j k_{+} x}+p^{-} \mathrm{e}^{-j k_{-} x} \\
& k_{+}=\frac{k}{1+M}, k_{-}=\frac{k}{1-M}
\end{aligned}
$$

Equation (2) presents superposition of two progressive waves moving in opposite directions.

When there is a side branch Helmholtz resonator along the flow duct as shown in Figure 1. A side-branch Helmholtz resonator is mounted on the side wall of an infinitely duct and the plane wave propagates along the duct. The cross-sectional area of the duct is $A$. The mean flow in the duct is $U_{0}$. The dotted area indicates the pressure discontinuity due to the mean flow. At the connection, the upstream pressure is $p_{1}$ and the downstream pressure is $p_{2}$. The pressure at the neck of the Helmholtz resonator is $p_{3}$ and is assumed to be equal to the upstream pressure at the junction.

At the junction, according to Equation (2) the sound pressure can be expressed as follows:

$$
\begin{aligned}
& p_{1}=\left(I \mathrm{e}^{-\mathrm{i} k_{I} x}+\mathrm{Re}^{\mathrm{i} k_{R} x}\right) \mathrm{e}^{\mathrm{i} \omega t} \\
& p_{2}=T \mathrm{e}^{\mathrm{i} k_{T} x} \mathrm{e}^{\mathrm{i} \omega t} \\
& k_{I}=\frac{\omega / c}{1+M}, k_{R}=\frac{\omega / c}{1-M}, k_{T}=\frac{\omega / c}{1+M} \\
& \longrightarrow U_{0} \rightleftarrows p_{1}
\end{aligned}
$$

Figure 1. A side branch Helmholtz resonator in a grazing flow duct.
The fluid particle velocities satisfy the following equation:

$$
\frac{D u_{1}}{D t}=-\frac{1}{\rho_{0}} \frac{\partial p_{1}}{\partial x}, \frac{D u_{2}}{D t}=-\frac{1}{\rho} \frac{\partial p_{2}}{\partial x}
$$

The propagation of the wave can be characterized by the following equations [13]:

$$
\begin{aligned}
& \left(P_{0}+p_{1}\right) A+\left(\rho_{0}+\rho_{1}\right)\left(U_{0}+u_{1}\right)^{2} A \\
& =\left(P_{0}+p_{2}\right) A+\left(\rho_{0}+\rho_{2}\right)\left(U_{0}+u_{2}\right)^{2} A \\
& \left(\rho_{0}+\rho_{1}\right)\left(U+u_{1}\right) A \\
& =\left(\rho_{0}+\rho_{2}\right)\left(U+u_{2}\right) A+\left(\rho_{0}+\rho_{3}\right) S_{n} u_{3}
\end{aligned}
$$

where $P_{0}$ is the equilibrium pressure, $U_{0}$ is the mean flow velocity and $\rho_{0}$ is the equilibrium density. Equation (7) and Equation (8) respectively indicates conservation of momentum and flow mass at the junction. Substituting Equations (1)-(5) into Equations (7) and (8)

$$
\begin{aligned}
& (1+M)^{2} I+(1-M)^{2} R=\left(1+M^{2}\right) T \\
& (1+M-C) I-(1+M+C) R=(1+M) T \\
& C=\rho c / A Z_{H R}
\end{aligned}
$$

$R$ is eliminated from the Equations (7)-(8). Thus, $T$ is indicated with $I$ as follows:

$$
\frac{T}{I}=\frac{2\left(-M^{2}+2 M C+1\right)}{(1+M)[(1+M) C+2(1-M)]}
$$

Equation (10) shows the relationship between transmission wave and incident wave in a grazing flow duct.

\section{Flow Effect on Acoustic Impedance of Helmholtz Resonator}

As shown in Equation (11), the transmission loss performance of the Helmholtz resonator is mainly determined by the acoustic impedance and Mach number. The acoustic impedance of Helmholtz resonator can be expressed as below:

$$
Z_{H R}=R_{H R}+j X_{H R}=R_{H R}+j\left(\omega M_{H R}-\frac{1}{\omega C_{H R}}\right)
$$

$R_{H R}$ and $X_{H R}$ are respectively the acoustic resistance and the acoustic reactance of the Helmholtz resonator. The resonance frequency $f_{0}=1 / \sqrt{M_{H R} C_{H R}}=\sqrt{S / l_{\text {eff }} V}$ of Helmholtz resonator is determined by the reactance. The acoustic reactance reaches minimum when resonance occurs.

A lot of studies have been conducted on the acoustics impedance of a HR in a grazing flow duct. Although there is no unified model to indicate the flow effect on 
acoustic impedance of the HR, it is agreed that the flow mainly influence the resistance and the effective length of Helmholtz resonator. Many experimental work shows that acoustic resistance increases linearly and the effective length of the neck decrease as the flow velocity increased. Cummings [14] proposed an empirical impedance model:

$$
\begin{aligned}
& \frac{R_{f} c_{0}}{f d}=\left(12.52\left(\frac{t}{d}\right)^{-0.32}-2.44\right)\left(\frac{u_{*}}{f d}\right)-3.2 \\
& \frac{\delta}{\delta_{0}}=1,\left(\frac{u_{*}}{f t} \leq \frac{d}{t}\right) \\
& \frac{\delta}{\delta_{0}}=\left(1+0.6 \frac{t}{d}\right) \exp \left[-\frac{\frac{u_{*}}{f t}-0.12 \frac{d}{t}}{0.25+\frac{t}{d}}\right]-0.6 \frac{t}{d}, \\
& \left(\frac{u_{*}}{f t}>\frac{d}{t}\right)
\end{aligned}
$$

$d$ and $f$ are respectively diameter and the length of the Helmholtz resonator. $R_{f}$ denotes resistance introduced by flow. $\delta$ is the end correction of the neck with grazing mean flow, $\delta_{0}$ is the end correction without flow. $u_{*}$ is friction velocity at the boundary surface. With Cummings' model, the effect of various flow speeds on a Helmholtz resonator is show in Figure 2.

In Figure 2, the geometries of the Helmholtz resonator are: cross sectional area of duct $S d=0.0144$, resonator orifice area $S n=0.0004$, length of the resonator neck is $L n=0.03 \mathrm{~m}$, the cavity of the resonator $V=0.0014$. From the result shown in Figure 2, the transmission loss is influenced in the items of the resonant frequency and the transmission loss amplitude at resonant frequency. As the mean flow speed increased, the resonance occurs at a higher frequency and the transmission loss performance decreased at the higher frequency.

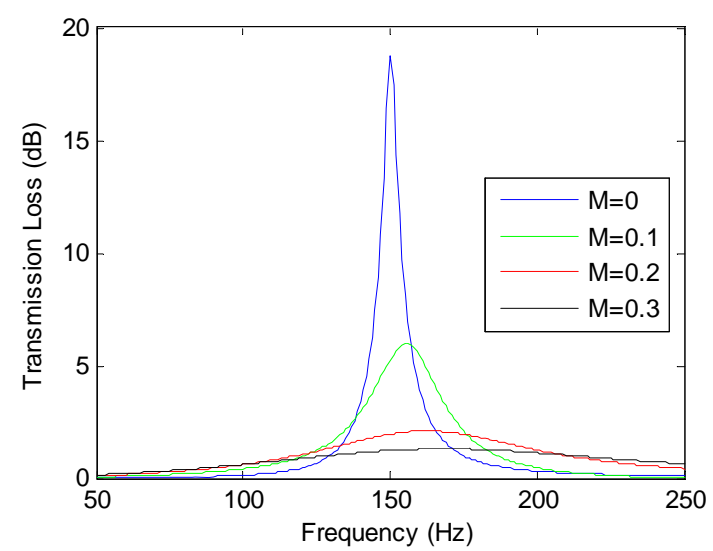

Figure 2. Transmission loss of a Helmholtz resonator under different flow condition.
In a practical exhaust ductwork system, the Mach number is normally less than 0.3 . In that case, the convective flow effects in the duct can be neglected [15]. The mainly important effect of mean flow is on the acoustic impedance of Helmholtz resonator. The transmission loss is below:

$$
T L=10 \log \left[\frac{\left(\rho c / 2 A+R_{H R}\right)^{2}+X_{H R}^{2}}{R_{H R}^{2}+X_{H R}^{2}}\right]
$$

This equation indicates that the transmission loss reach a peak when the reactance approaching to zero and the resistance significantly influence the sound reduction performance at the resonance frequency. At resonance frequency, the acoustic reactance is close to zero, therefore the transmission loss will be:

$$
T L=10 \log \left[\frac{\left(\rho c / 2 A+R_{H R}\right)^{2}}{R_{H R}^{2}}\right]
$$

Transmission loss will decrease as the resistance increases. It is known that acoustic resistance increases linearly and the effective length of the neck decrease as the flow velocity increased. Therefore, the flow influences the attenuation performance of Helmholtz resonator in two ways: the resistance at the orifice and the effective length of the neck. The decrease in length end correction results in resonance frequency shifts to a higher frequency and the increased resistance leads to lower transmission loss amplitude at resonance frequency.

\section{Termination Impedance Control}

This formula (11) indicates the discontinuity condition due to the mean flow and the flow will reduce the transmission loss performance of Helmholtz resonator. An emerging solution aims to adapt to the changes of the environment is semi active control system. Semi active control method using active noise control strategy adaptively changes the passive resonator to follow the environmental variation. However, controlling low frequency makes the system bulky and costly. The concept of semi-active control is to control noise by changing the acoustic impedance of Helmholtz resonator. Here we control the acoustic impedance of Helmholtz through controlling end termination impedance instead of physical geometries.

After the discontinuity condition where the main duct joins the side branch Helmholtz resonator is formulated, it is possible to consider reducing the noise via termination impedance control of the side branch Helmholtz resonator.

The system configuration is shown in Figure 3. The side Helmholtz resonator is ended by a control source. 
The termination impedance is controlled by detecting the sound pressure near the resonator neck and the controller function is defined as $H(\omega)$. The acoustic circuit of the semi active Helmholtz resonator is shown in Figure 4. The $R_{H R}, M_{H R}$, and $C_{H R}$ are the acoustic resistance, acoustic inertance, and acoustic compliance of the Helmholtz resonator under flow condition.

According to the acoustic circuit, the pressure $p_{3}$ can be readily expressed as:

$$
p_{3}=U_{3}\left(j \omega M_{H R}+R_{H R}\right)+\frac{U_{3}-H(\omega) p_{3}}{j \omega C_{H R}}
$$

Thus, the impedance of the semi active Helmholtz resonator is;

$$
Z_{3}=\frac{p_{3}}{U_{3}}=\frac{j \omega M_{H R}+R_{H R}+1 / j \omega C_{H R}}{1+H(\omega) / j \omega C_{H R}}
$$

Substituting Equation (19) to Equation (11) we can obtain the transmission coefficient with the termination impedance control:

$$
\frac{T}{I}=\frac{2\left[\frac{2 M \rho_{0} c_{0}}{A}+\left(1-M^{2}\right) Z_{3}\right]}{(1+M)\left[\frac{(1+M) \rho_{0} c_{0}}{A}+2(1-M) Z_{3}\right]}
$$

To suppress the transmitted wave means to let transmission coefficient approach to minimum. Thus, the controller function should be determined such that the Equation (12) equals zero. Thus, we obtain

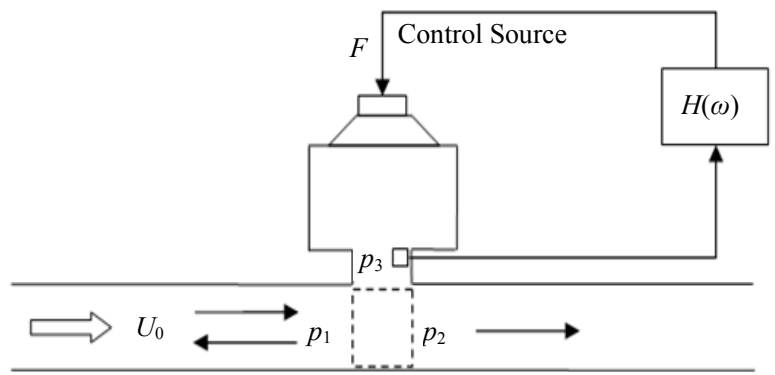

Figure 3. Control system model.

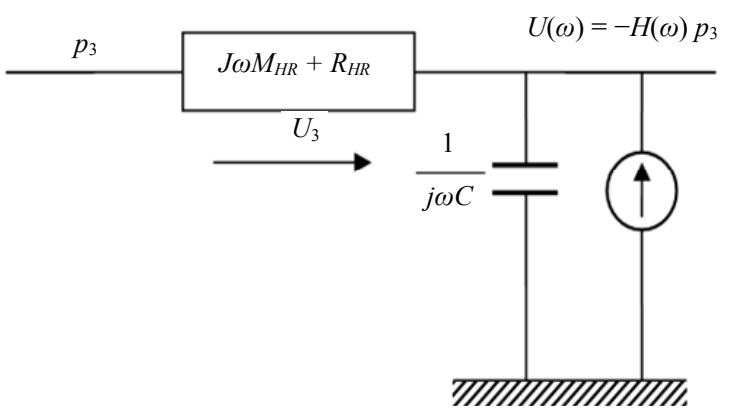

Figure 4. Acoustic circuit.

$$
\begin{aligned}
& H(\omega)= \\
& j \omega C_{H R}\left[-\frac{A\left(M^{2}-1\right) j \omega M_{H R}+R_{H R}+1 / j \omega C_{H R}}{2 M \rho_{0} c_{0}}-1\right]
\end{aligned}
$$

The controller function is related to impedance of the Helmholtz resonator and the mean flow velocity. Adjusting the controller function could reach maximum transmission loss over a wider frequency range. The mean flow significantly influences the acoustic impedance of the Helmholtz resonator and there is no a unified model to predict the effect of flow on the acoustic impedance of the Helmholtz resonator and it will be determined by investigating the lumped parameters under different air flow velocities experimentally.

\section{Conclusion}

Pressure discontinuity occurs when there is a mean flow in duct system. The transfer function at the joint area is analytically formulated based on the conservation of the momentum and mass of air flow. Termination impedance control is then considered to control the transmission noise under flow condition. The controller function is derived theoretically to reach to noise control over a wider frequency range in a low Mach flow duct.

\section{Acknowledgements}

The work described in this paper was fully supported by a grant from the Research Grants Council of the Hong Kong Special Administrative Region, China (Project No. PolyU 5263/09E).

\section{REFERENCES}

[1] J. S. Lamancusa, "An Actively Tuned, Passive Muffler System for Engine Silencing," INCE Conference Proceedings, Vol. 126, No. 1, 1987, pp. 313-318.

[2] T. Izumi and T. Narikiyo, "Muffler System Controlling the Volume of Resonant Cavity," The Journal of the Acoustical Society of Japan, Vol. 46, No. 6, 1990, pp. 479485.

[3] E. Esmailzadeh, A. Alasty and A. R. Ohadi, "Hybrid Active Noise Control of a One-Dimensional Acoustic Duct," Journal of Vibration and Acoustics-Transactions of the Asme, Vol. 124, No. 1, 2002, pp. 10-18. doi:10.1115/1.1400117

[4] H. Matsuhisa, B. S. Ren and S. Sato, "Semiactive Control of Duct Noise by a Volume-Variable Resonator," Jsme International Journal Series III-Vibration Control Engineering Engineering for Industry, Vol. 35, No. 2, 1992, pp. 223-228. doi:10.1299/jsmec 1988.35.223

[5] T. Izumi, H. Takami and T. Narikiyo, "Muffler System Controlling an Aperture Neck of a Resonator," Journal of the Acoustical Society of Japan, Vol. 47, No. 9, 1991, pp. 647-652. 
[6] C. Radcliffe and C. Birdsong, "An Electronically Tunable Resonator for Noise Control," Proceedings of the 2001 Noise and Vibration Conference, Michigan, 30 April-3 May 2001.

[7] M. Utsumi, "Reduction of Noise Transmission in a Duct by Termination Impedance Control of a Sidebranch Resonator," Journal of Vibration and Acoustics, Vol. 123, No. 3, 2001, pp. 289-296. doi:10.1115/1.1368118

[8] J. Yuan, "Active Helmholtz Resonator with Positive Real Impedance," Journal of Vibration and Acoustics-Transactions of the Asme, Vol. 129, No. 1, 2007, pp. 94-100. doi: $10.1115 / 1.2345678$

[9] E. Meyer, F. Mechel and G. Kurtze, "Experiments on the Influence of Flow on Sound Attenuation in Absorbing Ducts," Journal of the Acoustical Society of America, Vol. 30, No. 3, 2007, pp. 165-174. doi:10.1121/1.1909527

[10] J. S. Anderson, "The Effect of an Air Flow on a Single
Side Branch Helmholtz Resonator in a Circular Duct," Journal of Sound and Vibration, Vol. 52, No. 3, 1977, pp. 423-431. doi:10.1016/0022-460X(77)90569-7

[11] A. Hersch and B. Walker, "Effect of Grazing Flow on the Acoustic Impedance of Helmholtz Resonators Consisting of Single and Clustered Orifices," NASA Contractor Report $3177,1979$.

[12] K. U. Ingard, "Notes on Acoustics," Infinity Science Press, Hingham, 2008.

[13] P. O. A. L. Davies, "Practical Flow Duct Acoustics," Journal of Sound and Vibration, Vol. 124, No. 1, 1988, pp. 91-115. doi:10.1016/S0022-460X(88)81407-X

[14] A. Cummings, "The Effects of Grazing Turbulent PipeFlow on the Impedance of an Orifice," Acta Acustica United with Acustica, Vol. 61, No. 4, 1986, pp. 233-242.

[15] COMSOL 4.3a, Acoustic Module Model Library, 2012. 